\title{
PALB2 upregulation is associated with a poor prognosis in pancreatic ductal adenocarcinoma
}

\author{
OUYANG GE ${ }^{1 *}$, ANLE HUANG $^{2 *}$, XIN WANG $^{3}$, YULING CHEN $^{4,5}$, YUANCHUN YE $^{6,7}$ and LUTZ SCHOMBURG ${ }^{1}$ \\ ${ }^{1}$ Institute for Experimental Endocrinology, Charité-Universitätsmedizin Berlin, Corporate Member of Freie Universität Berlin, \\ Humboldt-Universität zu Berlin and Berlin Institute of Health, D-13353 Berlin, Germany; ${ }^{2}$ Department of \\ Gastrointestinal Surgery, Xiamen Cancer Hospital, The First Affiliated Hospital of Xiamen University, Xiamen, Fujian 361003, \\ P.R. China; ${ }^{3}$ Medical Department for Hematology and Oncology, Klinikum rechts der Isar, Technische University Munich, \\ D-81675 Munich; ${ }^{4}$ Department of Rheumatology and Clinical Immunology, Charité-Universitätsmedizin Berlin, \\ Corporate Member of Freie Universität Berlin, Humboldt-Universität zu Berlin, and Berlin Institute of Health; \\ ${ }^{5}$ German Rheumatism Research Centre Berlin, Leibniz Institute, D-10117 Berlin, Germany; ${ }^{6}$ Department of Gastroenterology, \\ Quanzhou First Hospital Affiliated to Fujian Medical University, Quanzhou, Fujian 362000, P.R. China; ${ }^{7}$ Department of \\ Hematology and Oncology, Benjamin Franklin Campus, Charité-Universitätsmedizin Berlin, Corporate Member of \\ Freie Universität Berlin, Humboldt-Universität zu Berlin and Berlin Institute of Health, D-12203 Berlin, Germany
}

Received July 16, 2020; Accepted December 10, 2020

DOI: $10.3892 / \mathrm{ol} .2021 .12485$

\begin{abstract}
During DNA repair, BRCA1 and BRCA2 interact with the tumor suppressor partner and localizer of BRCA2 (PALB2). PALB2 mutations are associated with an increased risk of breast and ovarian carcinoma, and upregulated PALB2 expression is associated with poor clinical outcomes. The present study investigated the role and prognostic value of PALB2 in pancreatic ductal adenocarcinoma (PDAC). PALB2 expression was inhibited using a small interfering RNA in PDAC cell lines, and the subsequent effects on cell proliferation and migration were investigated. Tissue microarrays from 157 patients undergoing a pancreaticoduodenectomy for PDAC were analyzed via immunohistochemistry, and PALB2 expression was compared with patient outcomes using
\end{abstract}

Correspondence to: Dr Yuanchun Ye, Department of Gastroenterology, Quanzhou First Hospital Affiliated to Fujian Medical University, 215 Wenling South Road, Fengze, Quanzhou, Fujian 362000, P.R. China

E-mail: aye1357@163.com

Professor Lutz Schomburg, Institute for Experimental Endocrinology, Charité-Universitätsmedizin Berlin, Corporate Member of Freie Universität Berlin, Humboldt-Universität zu Berlin and Berlin Institute of Health, Augustenburger Platz 1, D-13353 Berlin, Germany

E-mail: lutz.schomburg@charite.de

${ }^{*}$ Contributed equally

Key words: prognostic marker, partner and localizer of BRCA2, pancreatic ductal adenocarcinoma, cell migration, epithelial-mesenchymal transition
Kaplan-Meier curves and the multivariate Cox regression model. PALB2-knockdown in PDAC cells had little effect on cell proliferation, but significantly decreased cell migration. Relatively high PALB2 expression was observed in PDAC tissues compared with in peritumoral tissues. Overall survival (OS) was negatively associated with PALB2 expression. TNM stage and PALB2 expression were identified as independent prognostic factors associated with OS via multivariate analysis. Overall, the present study demonstrated that PDAC cell migration was dependent on PALB2, which was further supported by the finding that elevated PALB2 expression in PDAC tissues was associated with poor survival in patients with PDAC. Therefore, PALB2 may serve as a novel prognostic marker in PDAC, which may aid with the development of therapeutic strategies for the disease.

\section{Introduction}

Pancreatic ductal adenocarcinoma (PDAC) is a highly aggressive malignancy with an overall 5-year survival rate of $9 \%$ (1). Globally, PDAC-associated deaths are predicted to increase by $0.5 \%$ in 2030 (2). Due to the lack of specific symptoms for early diagnosis and a tendency to metastasize quickly, the majority of patients present with late stage PDAC at diagnosis (3). Consequently, even after pancreaticoduodenectomy, the 5-year survival rate remains $<20 \%$ (3). Additional diagnostic and prognostic markers are required to improve early detection, the assessment of disease activity and prognosis, and therapeutic decisions and monitoring, as well as for the development of novel treatment strategies.

Numerous biomarkers have been identified and developed to improve the early diagnosis of PDAC (4). Among the genetic factors, BRCA1 and BRCA2 are two well-characterized tumor suppressor genes implicated in gene transcription and DNA repair (5). Gene mutations in BRCA1 and BRCA2 are 
associated with breast, ovarian, colorectal and prostate cancer, and genetic variants in these genes have also been associated with the risk of PDAC $(6,7)$.

Partner and localizer of BRCA2 (PALB2) was first identified as a protein that co-localized with BRCA2 in the nucleus; PALB2 serves as a linker between BRCA1 and BRCA2 in the repair of DNA breaks (8). Mutations in PALB2 are responsible for Fanconi anemia complementation group $\mathrm{N}$ and are associated with childhood cancers, such as solid tumors of the kidney (Wilms' tumor), medulloblastoma and neuroblastoma $(9,10)$. Additionally, single nucleotide polymorphisms in the PALB2 gene have been associated with the risk of breast cancer $(11,12)$. The association among hereditary mutations in PALB2, PDAC risk and incidence rates exhibits population-specific characteristics (13-16). Compared with no or mild mutations (replacement of a single amino acid with a structurally related one), inactivating mutations in BRCA1, BRCA2 or PALB2 confer a more favorable prognosis in patients with PDAC (17), potentially due to higher genomic instability and an improved response to platinum-based chemotherapy (18-20). In addition, carbohydrate antigen 19-9 displays some prognostic value, but standardized predictive biomarkers to assess treatment success have not yet been identified (21).

The mRNA and protein expression levels of PALB2, as well as the role of PALB2 in PDAC tissues and its potential prognostic value, are not completely understood. Therefore, the present study investigated PALB2 expression in human PDAC cell lines and PDAC tumor and peritumoral tissue sections. Moreover, the association between PALB2 expression and disease characteristics or patient survival was further assessed in the present study.

\section{Materials and methods}

Patients and sample collection. Human PDAC tissue microarrays, as well as human breast and gastric cancer tissue microarrays (including 15 surgically resected breast/gastric tumor tissues), were obtained from Shanghai BioChip Co., Ltd. PDAC tissue microarray chips contained 157 tumor tissue samples (Table I) and 121 peritumoral tissues, which were free of signs of malignant transformation and distant from the tumor. The information on patient survival ranged from 1.2 to 7.0 years. TNM staging (7th edition) and American Joint Committee on Cancer staging data (7th edition) (22) were available for the majority of PDAC samples (the data were not available for 23 samples). Expression levels of tumor-associated markers, including Ki-67, p53, CD8 and programmed death ligand 1 (PDL1), were obtained from the database of the pathology department of the National Engineering Center of Shanghai BioChip Co., Ltd. All patients with PDAC were diagnosed by positive histology of invasive ductal carcinoma. Overall survival (OS) was calculated as the time interval between the date of surgery and the date of death or last follow-up visit. Not all deceased subjects were subjected to pathological analysis, and therefore the rate of PDAC-associated death is unknown.

Public data resource analysis. The level 3 information (aggregated, normalized and/or segmented data) of The Cancer Genome Atlas (TCGA, https://portal.gdc.cancer.gov/) containing pancreaticadenocarcinomadatasets of 178 individual tumors were downloaded for expression analysis (survival data were available for 177 patients). The Root Mean Squared Error normalized mRNA count ('count'), which represents PALB2 gene expression, was evaluated. For Gene Set Enrichment Analysis (GSEA), the latest official tool was downloaded from software.broadinstitute.org/gsea (version 3.0). The cBio Cancer Genomics Portal (cbioportal.org) was used to analyze PALB2 mRNA expression across different types of human cancer, based on TCGA public database.

Immunohistochemistry (IHC) and evaluation of immunostaining. Thin slices (4- $\mu$ m-thick sections) from $10 \%$ formalin-fixed ( $24 \mathrm{~h}$ at room temperature), paraffin-embedded tissue specimens were used. The sections were deparaffinized in xylene and rehydrated in a descending alcohol series $(100,95,90,75$ and $70 \%)$, using routine procedures. Antigen retrieval was achieved by digesting the sections with proteinase $\mathrm{K}\left(0.2 \mathrm{mg} / \mathrm{ml}\right.$ at $37^{\circ} \mathrm{C}$ for $\left.10 \mathrm{~min}\right)$ before $\mathrm{IHC}$, followed by repeated washing steps and blocking with $3 \%$ $\mathrm{H}_{2} \mathrm{O}_{2}$ for $30 \mathrm{~min}$ at room temperature. Sections were incubated with a rabbit polyclonal anti-PALB2 antibody $(1: 2,000$; cat. no. ab202970; Abcam) for $12 \mathrm{~h}$ at $4^{\circ} \mathrm{C}$ and then with an HRP-conjugated anti-rabbit secondary antibody (1:5,000; cat. no. P044801; Dako; Agilent Technologies, Inc.) for $30 \mathrm{~min}$ at room temperature. Tissue microarray slides were scanned using a Leica Aperio digital slide scanner (Leica Microsystems $\mathrm{GmbH}$ ). Tumor cells displaying staining in the nucleus were categorized as positively stained. The percentage of PALB2 ${ }^{+}$ tumor cells was calculated as the ratio of stained to unstained tumor cells. The percentages of positive cells were classified into five scores: $0,0 \% ; 1,1-5 \% ; 2,6-30 \% ; 3,31-60 \%$; and 4 , $61-100 \%$ positively stained cells. The scoring of each tissue section was conducted independently by two pathologists. In cases of inconsistent results ( $\sim 15 \%$ of samples), the pathologists re-evaluated the sample in question together to achieve a consensus. Tissue sections with scores $0-1$ were considered as low expression, whereas scores of 2-4 were considered as high expression. According to the information from TCGA, gastric cancer tissues are known to express low PALB2 protein levels, while breast cancer tissues are known for high PALB2 expression; therefore, the reliability of staining with the commercial PALB2 antibody was tested in a gastric cancer tissue as a negative control, and a breast cancer tissue as a positive control.

Cell lines, cell culture and small interfering (si)RNA. SW1990, PANC1 and CFPAC1 cell lines were purchased from The Cell Bank of Type Culture Collection of the Chinese Academy of Sciences. SW1990, PANC1 and CFPAC1 cells were maintained at $37^{\circ} \mathrm{C}$ with $5 \% \mathrm{CO}_{2}$ and cultured in DMEM supplemented with 10\% FBS (both Gibco; Thermo Fisher Scientific, Inc.). A siRNA targeting PALB2 (si-PALB2) and a scrambled negative control siRNA (si-NC) were purchased from Santa Cruz Biotechnology, Inc. (cat. nos. sc-93396 and sc-37007). Transfection was performed using standard protocols of Lipofectamine ${ }^{\circledR} 3000$ (Invitrogen; Thermo Fisher Scientific, Inc.). Lipofectamine 3000 reagent and siRNAs were diluted separately with OPTI-MEM (Gibco; Thermo Fisher Scientific, Inc.) in a centrifuge tube, then Lipofectamine 3000 and siRNAs were mixed and incubated for $15 \mathrm{~min}$ at room 
Table I. Clinicopathological characteristics of patients with PDAC $(n=157)$ and PALB2 protein expression in PDAC tissues.

\begin{tabular}{|c|c|c|c|}
\hline Features & PALB2 negative, $n=93$ & PALB2 positive, $\mathrm{n}=64$ & P-value \\
\hline Mean age $\pm S D$, years & $64.1 \pm 11.0$ & $62.6 \pm 10.1$ & 0.3851 \\
\hline Age range, years & $41-85$ & $34-81$ & \\
\hline Sex, n (\%) & & & 0.9325 \\
\hline Male & $56(58.3)$ & $40(41.7)$ & \\
\hline Female & $36(59.0)$ & $25(41.0)$ & \\
\hline T stage, $\mathrm{n}(\%)^{\mathrm{a}}$ & & & 0.0554 \\
\hline $\mathrm{T} 1-\mathrm{T} 2$ & $55(66.7)$ & $27(33.3)$ & \\
\hline $\mathrm{T} 3$ & $26(50.0)$ & $26(50.0)$ & \\
\hline $\mathrm{N}$ stage, $\mathrm{n}(\%)^{\mathrm{a}}$ & & & 0.4895 \\
\hline N0 & $47(63.5)$ & $27(36.5)$ & \\
\hline N1 & $34(57.6)$ & $25(42.4)$ & \\
\hline $\mathrm{AJCC}, \mathrm{n}(\%)^{\mathrm{a}}$ & & & 0.3948 \\
\hline $\mathrm{I}$ & $28(68.2)$ & $13(31.8)$ & \\
\hline IIA & $18(58.1)$ & $13(41.9)$ & \\
\hline IIB & $33(60.0)$ & $22(40.0)$ & \\
\hline IV & $2(33.3)$ & $4(66.7)$ & \\
\hline Tumor location & & & 0.6776 \\
\hline Head & $54(57.6)$ & $39(42.4)$ & \\
\hline Body/Tail & $39(60.9)$ & $25(39.1)$ & \\
\hline Ki-67 expression, $\mathrm{n}^{\mathrm{b}}$ & & & 0.3718 \\
\hline Positive & $47(75.8)$ & $15(24.2)$ & \\
\hline Negative & $18(66.7)$ & $9(33.3)$ & \\
\hline P53 expression, $\mathrm{n}^{\mathrm{b}}$ & & & 0.1810 \\
\hline Positive & $48(69.5)$ & $21(30.5)$ & \\
\hline Negative & $17(85.0)$ & $3(15.0)$ & \\
\hline PDL1 expression, $\mathrm{n}^{\mathrm{c}}$ & & & 0.1795 \\
\hline Positive & $19(48.7)$ & $20(51.3)$ & \\
\hline Negative & $5(29.4)$ & $12(70.6)$ & \\
\hline CD8 expression, $\mathrm{n}^{\mathrm{c}}$ & & & 0.8460 \\
\hline Positive & $19(42.2)$ & $26(57.8)$ & \\
\hline Negative & $5(45.5)$ & $6(54.5)$ & \\
\hline
\end{tabular}

${ }^{a} n=134 .{ }^{b} n=89 .{ }^{c} n=56$. T, primary tumor site; $N$, regional lymph node involvement; PDAC, pancreatic ductal adenocarcinoma; PALB2, partner and localizer of BRCA2; PDL1, programmed death ligand 1; AJCC, American Joint Committee on Cancer.

temperature. Subsequently, DNA-lipid complex was added to the cells and incubated for $48 \mathrm{~h}$ at $37^{\circ} \mathrm{C}$. The transfected cells were used for subsequent experiments.

Cell proliferation and migration via wound healing assay. Cell proliferation was assessed by performing the Cell Counting Kit-8 assay (Dojindo Molecular Technologies, Inc.). Briefly, cells were seeded into 96-well plates. After 0,24 or $48 \mathrm{~h}$, the number of viable cells was quantified after incubation with the CCK-solution for $2 \mathrm{~h}$ by measuring the optical density at a wavelength of $450 \mathrm{~nm}$ using a microplate reader. For the wound healing assay, cells were seeded into 6-well plates and transfected with si-PALB2 or si-NC. At $24 \mathrm{~h}$ post-transfection, a scratch wound was made in the confluent cell monolayer using a $200-\mu 1$ pipette tip. Cells were cultured in serum-free medium. The wound was observed at 0 and $48 \mathrm{~h}$ with a light microscope (x100 magnification) after scratching. Cell migration area was measured using ImageJ (v1.52q; National Institutes of Health).

Reverse transcription-quantitative PCR (RT-qPCR). Total RNA from cells was isolated using TRIzol ${ }^{\circledR}$ (Invitrogen; Thermo Fisher Scientific, Inc.). Total RNA (1 $\mu \mathrm{g})$ was reverse transcribed into cDNA using a ReverTra Ace qPCR RT kit (Toyobo Life Science) according to the manufacturer's protocol. Subsequently, qPCR was performed using SYBR Green Gene Expression Assays (Applied Biosystems; Thermo Fisher Scientific, Inc.) and an AB7500 Real-Time PCR System (Applied Biosystems; Thermo Fisher Scientific, Inc.). Thermocycling conditions included denaturation at $95^{\circ} \mathrm{C}$ for 
$15 \mathrm{sec}$, followed by annealing at $60^{\circ} \mathrm{C}$ for $10 \mathrm{sec}$ and elongation at $72^{\circ} \mathrm{C}$ for $20 \mathrm{sec}$, for 40 cycles. The following primers were used for qPCR: Snail family transcriptional repressor 1 (Snail) forward, 5'-ACCACTATGCCGCGCTCTT-3' and reverse, 5'-GGTCGTAGGGCTGCTGGAA-3'; snail family transcriptional repressor 2 (Slug) forward, 5'-ATGAGGAATCTGGCT GCTGT-3' and reverse, 5'-CAGGAGAAAATGCCTTTG GA-3'; zinc finger E-box binding homeobox 1 (Zeb1) forward, 5'-GCACCTGAAGAGGACCAGAG-3' and reverse, 5'-TGC ATCTGGTGTTCCATTTT-3'; Vimentin forward, 5'-GAC GCCATCAACACCGAGTT-3' and reverse, 5'-CTTTGTCGT TGGTTAGCTGGT-3'; and GAPDH forward, 5'-ACCACA GTCCATGCCATCAC-3' and reverse, 5'-TCCACCACCCTG TTGCTGTA-3'. mRNA expression levels were quantified using the $2^{-\triangle \triangle C q}$ method (23) and normalized to the internal reference gene GAPDH.

Western blotting. Total protein was extracted from pancreatic cancer cell lines using RIPA lysis buffer (cat. no. 89900; Thermo Fisher Scientific, Inc.). Protein concentration was measured using BCA kit and western blotting was performed according to standard protocols. Briefly, $30 \mu \mathrm{g}$ protein/lane was separated via $8 \%$ SDS-PAGE and transferred to a PVDF membrane. The membrane was blocked with 5\% BSA (cat. no. 9998; Cell Signaling Technology, Inc.) for $2 \mathrm{~h}$ at room temperature. Subsequently, the membrane was incubated with the following primary antibodies: Anti-PALB2 (1:1,000; cat. no. ab202970; Abcam) and anti- $\beta$-actin $(1: 2,000$; cat. no. sc-130656; Santa Cruz Biotechnology, Inc.) for $12 \mathrm{~h}$ at $4{ }^{\circ} \mathrm{C}$. A mouse anti-rabbit IgG HRP-conjugated secondary antibody (1:2,000; cat. no. sc-2357; Santa Cruz Biotechnology, Inc.) was used and the membrane was incubated for $30 \mathrm{~min}$ at room temperature. TBS-Tween $(0.5 \%$ Tween) was used for membrane washing, and an ECL kit (Bio-Rad Laboratories, Inc.) was used for visualization. Protein expression levels were semi-quantified using ImageJ software v1.52q (National Institutes of Health) with $\beta$-actin as the loading control.

Statistical analysis. Statistical analyses were performed using GraphPad Prism (v8.0; GraphPad Software, Inc.) or SPSS (v24.0; IBM Corp.) softwares. Continuous variables were compared using the unpaired Student's t-test or Mann-Whitney $\mathrm{U}$ test. The $\chi^{2}$ test was used to analyze the distribution of categorical variables between PALB2- and PALB2 ${ }^{+}$groups. OS was plotted as a Kaplan-Meier survival curve with $95 \%$ CIs, and differences between subgroups were compared using log-rank tests. Cox regression analysis was performed to identify independent prognostic factors. $\mathrm{P}<0.05$ was considered to indicate a statistically significant difference.

\section{Results}

PALB2 IHC. To establish the IHC method and test whether the selected antibody selected was capable of yielding congruent data in agreement with current knowledge, stomach and breast carcinoma tissues were measured for PALB2 expression as negative and positive controls, respectively. Weak staining signals were observed for the negative control stomach cancer tissue (Fig. S1; upper panels), while strong staining signals were observed for the positive control breast carcinoma tissues

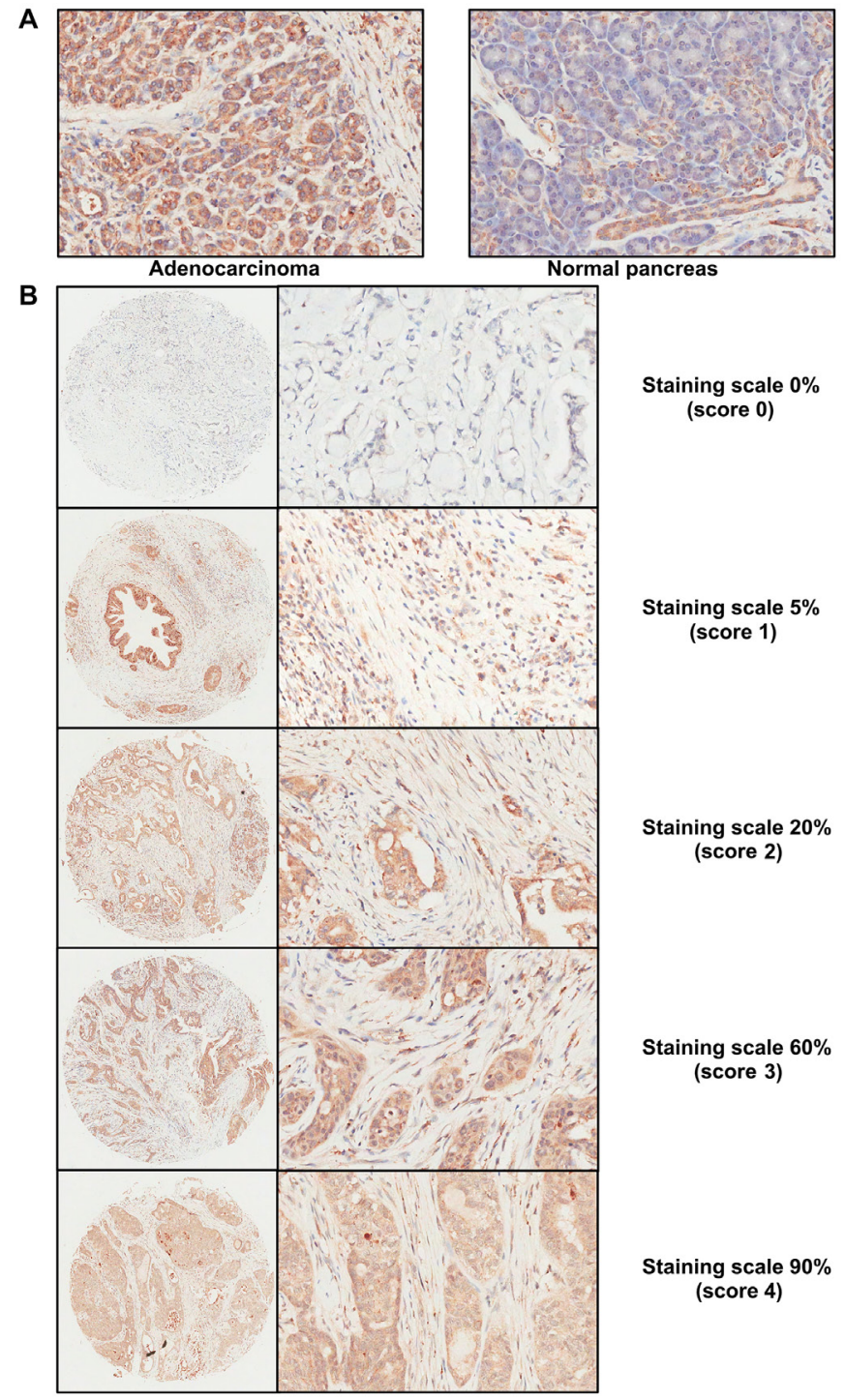

Figure 1. Immunohistochemistry for PALB2 expression. (A) PALB2 staining was stronger in pancreatic ductal adenocarcinoma tissues compared with in para-tumoral normal tissues (magnification, x400). (B) PALB2 expression was scored 0-4 according to the percentage of positively stained nuclei. Representative images of each score are shown, along with magnified tissue areas (magnification, x80 and 400, respectively). PALB2, partner and localizer of BRCA2.

(Fig. S1; lower panels). The results supported the suitability of the antibody and the IHC protocol, and the staining pattern was consistent with the results obtained using the public TCGA database.

PALB2 expression in PDAC tissues. Subsequently, PALB2 expression in PDAC samples was analyzed using characterized tissue samples arranged in an array format. The staining patterns were evaluated by two independent pathologists and a consensus was reached on the resulting data. The number of PALB2- samples was slightly higher compared with that of $\mathrm{PALB}^{+}$samples within the array of 157 tumor samples analyzed (Table I). In PALB2 ${ }^{+}$samples, compared with in the para-tumor normal tissues, staining was stronger in PDAC tissues (Fig. 1A). PALB2 ${ }^{+}$cell nuclei in PDAC samples and peritumoral tissues were identified and counted in relation to unlabeled cells. Overall, the positive rates for PALB2 were 

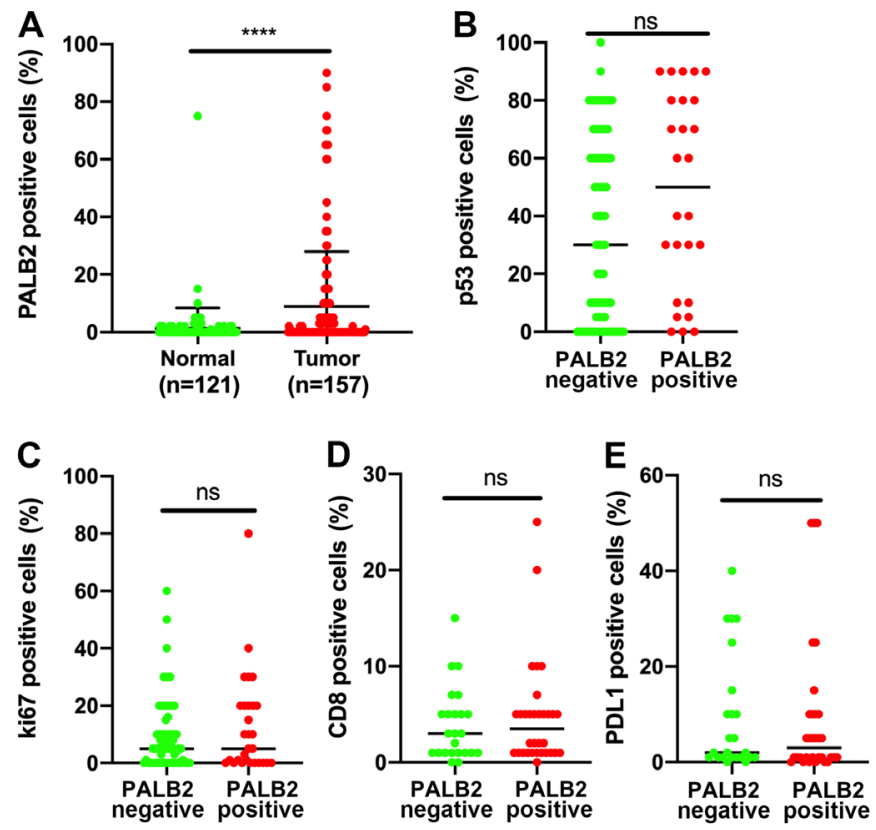

Figure 2. Association between PALB2 expression and the classical markers p53 and Ki-67, as well as the cancer immune markers PDL1 and CD8. (A) Relative number of PALB2 positively stained cells was significantly higher in the carcinoma tissues compared with in peritumoral tissues. There was no association between the expression levels of (B) p53 or (C) Ki-67 and PALB2. There was no association between the expression levels of (D) CD8 or (E) PDL1 and PALB2. The groups were compared using the non-parametric Mann Whitney U test. ${ }^{* * * * *} \mathrm{P}<0.0001$. ns, not significant; PDL1, programmed death ligand 1; PALB2, partner and localizer of BRCA2.

$24.8 \%$ in para-tumor healthy tissues and $40.8 \%$ in PDAC tissues. The distribution of the samples with respect to the IHC score of 0-4 for the PDAC and peritumor tissue samples are presented in Table SI. Representative images of PALB2 IHC labeling for each score are shown in Fig. 1B.

Association between PALB2 and classical cancer markers. To assess whether PALB2 expression was associated with classical cancer markers, the expression pattern of PALB2 expression in tumor and peritumoral tissues was assessed. The results indicated that PALB2 expression was significantly lower in peritumoral tissues compared with in PDAC tissues (Fig. 2A). A direct comparison of Ki-67, p53, CD8 and PDL1 staining patterns in tissues with positive or negative PALB2 expression indicated no significant differences, suggesting that the expression levels of Ki-67, p53, CD8 and PDL1 were independent from PALB2 expression (Fig. 2B-E),

Association of PALB2 and other parameters with OS in patients with PDAC. The association between patient clinical characteristics, including tumor PALB2 expression, and OS was analyzed in 157 patients. Multivariate and univariate Cox proportional hazard regression analyses identified that $\mathrm{N}$ stage, age and PALB2 expression were significantly associated with OS in patients with PDAC (Table II). Kaplan-Meier analysis revealed that patients with positive PALB2 expression had a poorer OS rate compared with patients with negative PALB2 expression ( $\mathrm{P}=0.0384$; Fig. 3A). Furthermore, patients were divided into two groups: Low PALB2 expression (positive rate $55 \% ; n=119$ ) and high PALB2 expression (positive rate
$>5 \%$; $n=38$ ). Kaplan-Meier curves demonstrated a significant negative association between high PALB2 expression and OS $(\mathrm{P}=0.0195$; Fig. 3B). Therefore, high PALB2 expression may indicate a poor prognosis in patients with surgically resectable PDAC. By contrast, co-expression of p53 and PALB2 was not significantly associated with survival ( $P=0.1260$; Fig. 3C), whereas co-expression of PALB2 and Ki-67 indicated a poor prognosis in patients with PDAC $(\mathrm{P}=0.0415$; Fig. 3D). In addition, co-expression of PALB2 and the cancer immune markers CD8 and PDL1 were not associated with survival $(\mathrm{P}=0.1497$; Fig. 3E and $\mathrm{P}=0.5971$; Fig. 3F).

Relative PALB2 gene expression across different types of cancer. To investigate the spectrum of cancers associated with PALB2 expression on a larger scale, TCGA public database was analyzed. The mRNA expression levels of PALB2 in each type of cancer compared with their respective para-tumor tissues were presented in the log scale format (Fig. 4A). Most types of cancer in the database displayed upregulated PALB2 mRNA expression, including breast, cervical and pancreatic cancer, which is consistent with the detection of PALB2 protein expression in the present study. The 177 patients with PDAC from TCGA database were divided into two groups: Relatively high PALB2 expression group $(n=88)$ and relatively low PALB2 expression group ( $\mathrm{n}=89)$. The Kaplan-Meier curves demonstrated a significant negative association between high PALB2 expression and long-term survival rate $(\mathrm{P}<0.001$; Fig. 4B). Multivariate Cox regression analysis were conducted on the PALB2 mRNA expression data extracted from TCGA database (Table II). The multivariate analysis identified tumor location, $\mathrm{N}$ stage and PALB2 mRNA expression as tumor parameters with a significant association with OS (Table II). The summary of clinicopathological information of patients with PDAC from TCGA database are shown in Table SII.

Functional analysis of PALB2 in PDAC cells in vitro. To investigate the potential biological role of PALB2 in PDAC, PALB2 expression was knocked down using a siRNA in PDAC cells. The western blotting results indicated successful PALB2-knockdown by si-PALB2 compared with si-NC in SW1990 PDAC cells (Fig. 5A). Cell proliferation assays indicated similar proliferation rates in SW1990 cells regardless of PALB2-knockdown (Fig. 5B). Migration was assessed by performing wound healing experiments. Cell migration area was measured using ImageJ software. The results indicated that PALB2-knockdown inhibited PDAC cell migration depending on cell line. Migration was significantly decreased by PALB2-knockdown in SW1990 cells and CFPAC1 cells, but it was independent of PALB2 expression in PANC1 cells. (Figs. 5C and S2). Additionally, PALB2-knockdown significantly decreased the cell proliferation rate of CFPAC1 cells, but had no effect on PANC1 cells (Fig. S2).

Identification of biological signaling pathways associated with PALB2 expression. To identify biological signaling pathways associated with PALB2 expression, a GSEA was conducted on TCGA gene expression datasets from patients with different levels of PALB2 mRNA expression. Among the 189 available datasets, the top and bottom 20 datasets were selected. The most significantly enriched signaling pathways 
Table II. Multivariate Cox regression analyses of the overall survival of 157 patients with PDAC and PDAC samples represented in the TCGA database (May 2019).

A, Patients with PDAC $(n=157)$

\begin{tabular}{|c|c|c|c|c|}
\hline \multirow[b]{2}{*}{ Factors } & \multicolumn{2}{|c|}{ Multivariate analysis } & \multicolumn{2}{|c|}{ Univariate analysis } \\
\hline & HR $(95 \% \mathrm{CI})$ & P-value & $\mathrm{HR}(95 \% \mathrm{CI})$ & P-value \\
\hline Sex (Male/Female) & $1.375(0.913-2.071)$ & 0.128 & $1.182(0.816-1.712)$ & 0.377 \\
\hline Age ( $\geq 64 /<64$ years) & $0.645(0.423-0.982)$ & $0.041^{\mathrm{a}}$ & $0.773(0.541-1.106)$ & 0.159 \\
\hline Tumor location (head and body/tail) & $1.129(0.731-1.744)$ & 0.584 & $1.172(0.813-1.689)$ & 0.395 \\
\hline T classification(T1 and 2/T3) & $1.097(0.722-1.665)$ & 0.665 & $1.077(0.730-1.589)$ & 0.708 \\
\hline $\mathrm{N}$ classification $(\mathrm{N} 0 / \mathrm{N} 1)$ & $2.267(1.480-3.472)$ & $<0.001^{\mathrm{a}}$ & $1.720(1.182-2.504)$ & $0.005^{\mathrm{a}}$ \\
\hline PALB2 (high/low expression) & $1.789(1.142-2.803)$ & $0.011^{\mathrm{a}}$ & $1.598(1.068-2.389)$ & $0.022^{\mathrm{a}}$ \\
\hline
\end{tabular}

B, TCGA PDAC samples $(\mathrm{n}=177)$

\begin{tabular}{|c|c|c|c|c|}
\hline \multirow[b]{2}{*}{ Factors } & \multicolumn{2}{|c|}{ Multivariate analysis } & \multicolumn{2}{|c|}{ Univariate analysis } \\
\hline & HR $(95 \% \mathrm{CI})$ & P-value & HR $(95 \% \mathrm{CI})$ & P-value \\
\hline Sex (Male/Female) & $0.963(0.548-1.693)$ & 0.896 & $0.868(0.577-1.306)$ & 0.497 \\
\hline Age $(\geq 65 /<65$ years $)$ & $1.005(0.549-1.841)$ & 0.986 & $1.084(0.698-1.684)$ & 0.720 \\
\hline Tumor location (head and body/tail) & $1.970(1.083-3.585)$ & $0.026^{\mathrm{a}}$ & $2.108(1.170-3.801)$ & $0.013^{\mathrm{a}}$ \\
\hline $\mathrm{T}$ classification (T1 and $2 / \mathrm{T} 3$ and 4$)$ & $1.032(0.508-2.097)$ & 0.930 & $2.052(1.088-3.870)$ & $0.026^{\mathrm{a}}$ \\
\hline $\mathrm{N}$ classification $(\mathrm{N} 0 / \mathrm{N} 1)$ & $1.894(1.109-3.234)$ & $0.019^{\mathrm{a}}$ & $2.048(1.243-3.374)$ & $0.005^{\mathrm{a}}$ \\
\hline PALB2 (high/low expression) & $1.980(1.245-3.249)$ & $0.004^{\mathrm{a}}$ & $1.996(1.302-3.060)$ & $0.002^{\mathrm{a}}$ \\
\hline
\end{tabular}

${ }^{\mathrm{a}} \mathrm{P}<0.05$. T, primary tumor site; $\mathrm{N}$, regional lymph node involvement; TCGA, The Cancer Genome Atlas; PDAC, pancreatic ductal adenocarcinoma; HR, hazard ratio.

were selected based on the normalized enrichment scores. The results indicated that samples with relatively high PALB2 mRNA expression were enriched for genes associated with the 'epithelial mesenchymal transition' (EMT) signaling pathway, along with genes involved in the TNF- $\alpha$, TGF- $\beta$, p53, NOTCH and mTORC1 signaling pathways (Fig. 5D). To further validate the GSEA results, the expression levels of characteristic EMT-associated genes were quantified via RT-qPCR in PALB2-knockdown PDAC cells in vitro. SW1990 cells displayed PALB2-dependent migration effects. PALB2-knockdown significantly decreased Slug, Zeb1 and vimentin gene expression, but induced a small but non-significant increase on Snail expression. (Fig. 5E).

\section{Discussion}

In the present study, PALB2 expression in PDAC and peritumoral tissue samples was analyzed, and the effect of PALB2 on PDAC cell proliferation and migration in vitro was assessed. PALB2 protein expression was increased in tumor tissues compared with surrounding healthy tissues, and PALB2 mRNA expression levels were relatively high in PDAC compared with in other types of cancer. Upregulated PALB2 expression was not observed in stomach cancer in TCGA database and in the present IHC analysis. In PDAC, high mRNA and protein expression levels of PALB2 were negatively associated with OS, suggesting a potential positive effect of PALB2 on tumor cell migration and EMT signaling pathway-associated genes. The results suggested that elevated PALB2 expression may represent a diagnostic and prognostic marker in PDAC. Furthermore, $\mathrm{N}$ classification and age were significantly associated with OS in patients with PDAC; therefore, PALB2 expression, $\mathrm{N}$ classification and age may be combined to obtain a reliable marker for mortality risk. As PDAC is a rising and leading cause of cancer-associated mortality, additional markers are required to improve early and specific diagnosis of PDAC, and to aid with the development of treatment strategies (24).

The molecular mechanisms underlying the role of PALB2 in tumorigenesis are not completely understood (25). PALB2 co-localizes with BRCA1 and BRCA2, and contributes to error-free homologous recombination repair (26). Dysfunctions in BRCA1 and BRCA2 promote carcinogenesis (27). Upregulated BRCA2 expression predicts a poor prognosis in patients with breast carcinoma (28). Similarly, elevated PALB2 expression is associated with poor clinical outcomes in patients with advanced breast carcinoma (29). However, independent research has indicated no association between PALB2 expression and breast cancer prognosis (30), and therefore additional studies are required to clarify this interaction. PALB2 mutations are frequently described for some types of tumor, such as lung (31), breast and ovarian 
A
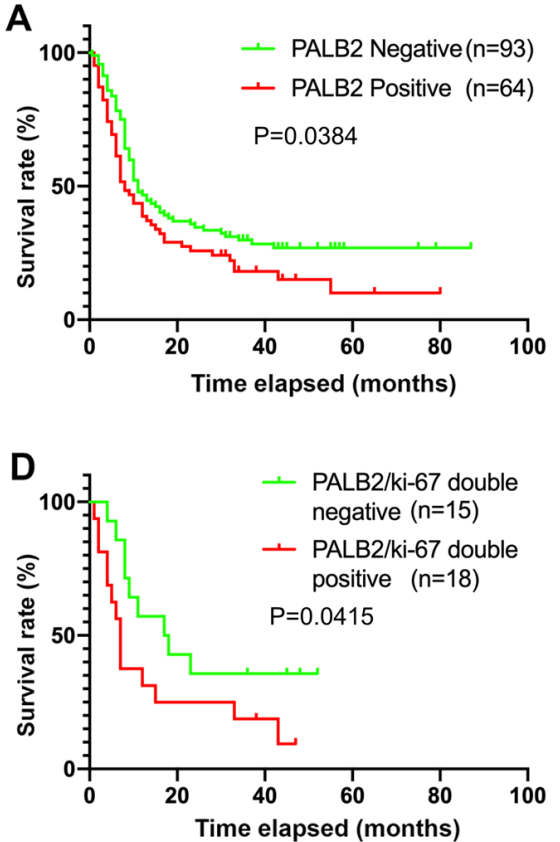

B
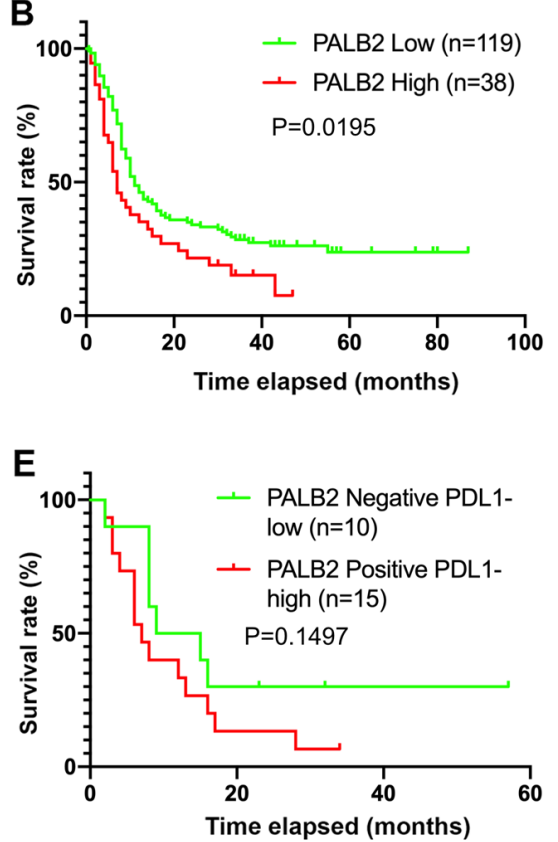
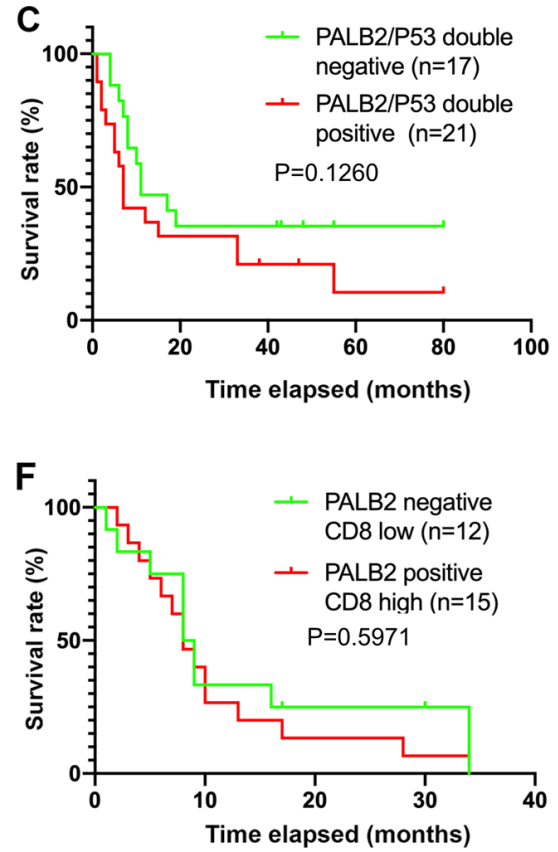

Figure 3. Kaplan-Meier estimates of OS according to cancer biomarker expression in patients with resectable PDAC. (A) The PALB2-positive PDAC group exhibited a worse OS rate compared with the PALB2-negative group. (B) Patients with high PALB2 expression exhibited a shorter OS rate compared with those with low PALB2 expression. (C) Co-expression of PALB2 and p53 had little effect on the OS of patients with PDAC. (D) The PALB2/Ki-67 double-positive PDAC group had a relatively poor OS compared with the group negative for PALB2 and Ki-67 protein expression. Co-expression of PALB2 and (E) PDL1 or (F) CD8 was not associated with OS of patients with PDAC. PDAC, pancreatic ductal adenocarcinoma; OS, overall survival; PDL1, programmed death ligand 1; PALB2, partner and localizer of BRCA2.

A Low

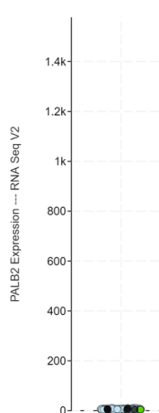

High

B

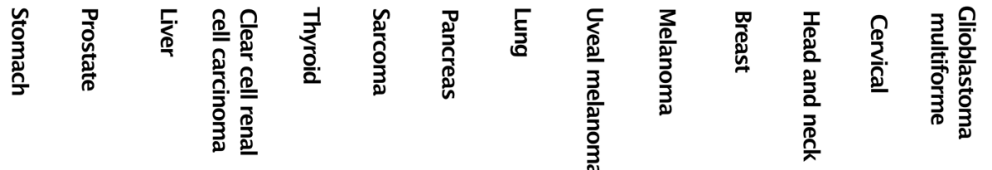

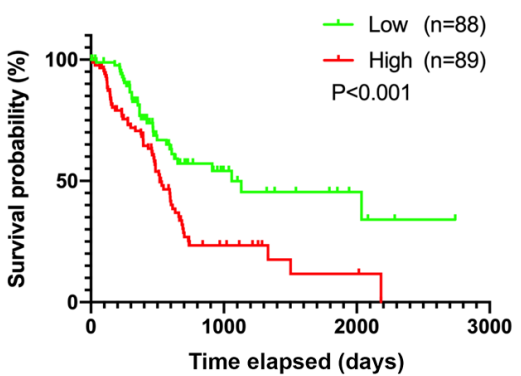

- Fusion • Truncating (Driver) • Missense(VUS) • Not mutated o Not profiled for mutations

Figure 4. PALB2 expression in 14 types of human cancer and predicted poor prognosis in PDAC. (A) Ascending scatter plots of PALB2 mRNA expression in each type of cancer were sorted by median PALB2 expression compared with the para-tumor normal tissues. (B) Separation of the patients with PDAC ( $\mathrm{n}=177$ ) from The Cancer Genome Atlas database by median PALB2 mRNA expression indicated that the low PALB2 expression group had an improved overall survival rate. PALB2, partner and localizer of BRCA2.

cancer (32). PALB2 mutations have been reported to predispose men and women to breast cancer (33), with the highest risk resulting from protein truncation mutations (34).

Missense polymorphisms in BRCA1 exert a moderate effect on the prognosis of patients with PDAC (35). Certain mutations in other BRCA signaling pathway genes predict an improved prognosis in patients with PDAC (17). Germline truncating mutations in PALB2 have been detected in patients with PDAC, indicating that PALB2 may serve as a potential susceptibility gene for pancreatic tumorigenesis (36). Another study suggested that truncating mutations of PALB2 may predispose individuals to breast carcinoma, as well as pancreatic carcinoma (37). Although the association between PALB2 and tumorigenesis has been reported, the relative importance of PALB2 dysregulation for the course of the disease, mortality risk and tumor cell characteristics are not completely understood. The identified role of PALB2 in PDAC cell migration is consistent with the inverse association between PALB2 
A

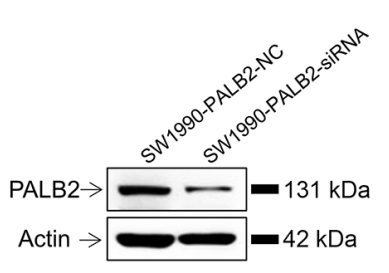

B

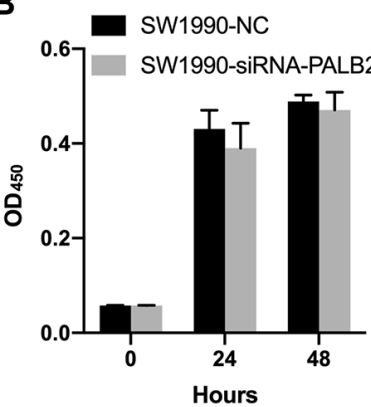

D

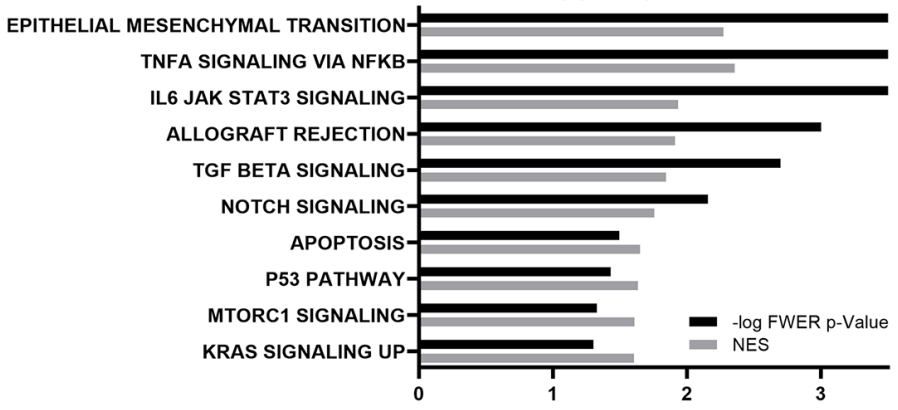

C Wound healing test

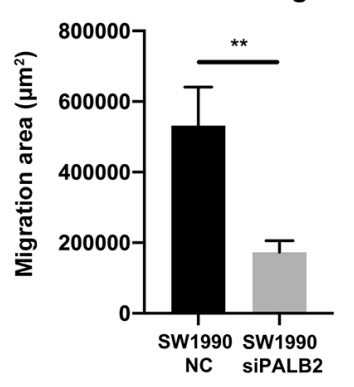

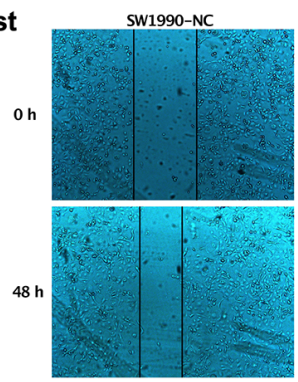

E

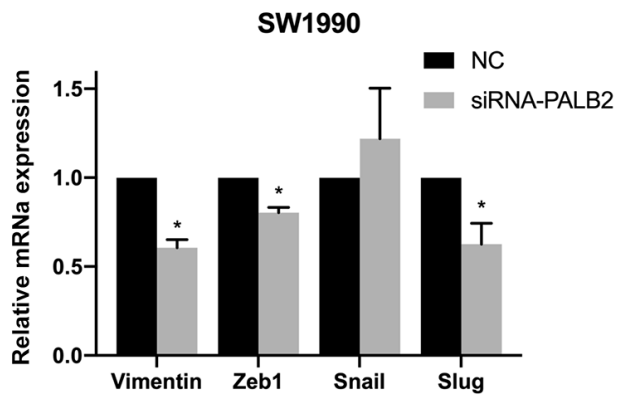

Figure 5. Inhibition of PALB2 expression in pancreatic ductal adenocarcinoma cells. (A) Western blot analysis of PALB2 expression indicated successful PALB2-knockdown by siRNA in SW1990 cells. (B) Cell proliferation rates were not affected by PALB2-knockdown. (C) Migration analysis of cells with PALB2-knockdown was determined by a wound healing assay and computer-assisted picture analysis (magnification, x100). (D) GSEA analysis identified 10 signaling pathways associated with PALB2 expression. (E) Reverse transcription-quantitative PCR analysis of EMT-associated genes following PALB2-knockdown in SW1990 cells. Biological triplicates were analyzed using an unpaired Student's t-test. " $\mathrm{P}<0.05$; ${ }^{* *} \mathrm{P}<0.01$. OD, optical density; NC, negative control; siRNA, small interfering RNA; PALB2, partner and localizer of BRCA2; NES, normalized enrichment score; GSEA, Gene Set Enrichment Analysis.

expression and OS observed in the present study. Therefore, it may be hypothesized that elevated PALB2 expression may be consistent with improved protection against DNA damage and low mutation rates in tumor cells as part of the BRCA-complex without conferring an increased risk of cancer-associated mortality. However, the aforementioned hypothesis was not in accordance with the present data, and it remains to be analyzed whether metastases are characterized by particularly high PALB2 expression supporting migration, and relatively low mutation rates compared with primary PDAC cells, potentially suggesting new therapeutic approaches for PDAC treatment and metastasis control (38). Furthermore, the integrity of the PALB2 gene, and whether overexpressed PALB2 is functional or mutated, requires further investigation.

The results of the present study indicated the potential prognostic significance of PALB2 expression in PDAC. The Kaplan-Meier analysis identified a strong association between PALB2 expression and poor OS. PALB2 expression was independent from other clinicopathological parameters, including Ki-67, p53, CD8 and PDL1 expression. Therefore, the results of the present study suggested that PALB2 may serve as an independent prognostic factor of OS in patients with surgically resectable PDAC and may be an additional biomarker to the classical parameters of PDAC staging (39).

In the present study, the in vitro experiments demonstrated that cell migration, but not cell proliferation, was altered by PALB2-knockdown. The corresponding GSEA highlighted several signaling pathways associated with PALB2, including EMT, TNF- $\alpha$, TGF- $\beta$, p53, NOTCH and mTORC1. As EMT was the most prominent signaling pathway associated with decreased OS, the expression levels of certain EMT genes in PDAC cells were assessed. Consistent with the GSEA results, PALB2-knockdown affected the expression levels of Slug, Zeb1 and vimentin, which was also consistent with a previous study (29). However, the extent to which the other significantly associated signaling pathways contribute to the decreased OS of patients with high PALB2 expression requires further investigation. PALB2 mutations are frequently observed in tumor metastases, as indicated by comparisons between localized and metastasized breast and prostate cancer, respectively $(40,41)$. Therefore, comparing sequence and expression level information on localized and metastasized PDAC should be conducted in future studies to further identify the potential functional role of PDAC.

In the present study, the association between PALB2 expression and OS was relatively strong. The present study used a relatively large sample size to identify and characterize the association between PALB2 expression and OS. However, the present study had a number of limitations. For example, as the study was a retrospective study, not all data on patients with PDAC and their treatments were available. Due to the observational type of the present study, mechanistic explanation could not be drawn, and the potential impact of varying expression levels of PALB2 on DNA damage was not studied. Additional functional studies are required to elucidate the biological role of elevated PALB2 protein expression in tumor cells and to determine the effect of PALB2 on cell proliferation, migration and tumor susceptibility to oncostatic medication, especially in association with the integrity of the gene. In conclusion, the present study demonstrated that 
PALB2 expression was increased in PDAC tissues, and an association between high PALB2 mRNA or protein expression and decreased OS was identified. The results suggested that PALB2 may serve as an additional diagnostic marker and may aid in predicting the risk of mortality in patients with PDAC. Moreover, the present results may aid in the management of patients in clinical practice and potentially in the development of personalized treatment strategies.

\section{Acknowledgements}

The authors would like to thank Dr Waldemar Minich and Dr Eddy Rijntjes (Charité-Universitätsmedizin Berlin, Berlin, Germany) for constructive discussions.

\section{Funding}

The present study was supported by Deutsche Forschungsgemeinschaft (Research Unit 2558 TraceAge, Scho 849/6-1). OG received a stipend from the China National Scholarship for Abroad.

\section{Availability of data and materials}

The datasets used and/or analyzed during the current study are available from the corresponding author on reasonable request.

\section{Authors' contributions}

OG and LS were responsible for the conceptualization of the study. OG and XW conducted the in silico analysis of The Cancer Genome Atlas database. YC and YY collected and translated patients' clinical data, and identified and selected the tissue samples for immunohistochemistry. OG and $\mathrm{AH}$ conducted the in vitro experiments. OG, YC and XW formally analyzed the data and conducted the statistical analyses. YY and LS supervised the project. OG, XW, YC, YY and LS contributed to drafting and writing the final manuscript. All authors read and approved the final manuscript. OG, YY and LS confirm the authenticity of all the raw data.

\section{Ethics approval and consent to participate}

The use of the pancreas, gastric and breast tumor tissues was approved by the Research Ethics Committees of the National Engineering Center of Shanghai BioChip Co., Ltd. The research has been performed according to the ethical standards of the Declaration of Helsinki. Oral informed consent was obtained from all patients prior to analysis according to the committee's regulations.

\section{Patient consent for publication}

Not applicable.

\section{Competing interests}

The authors declare that they have no competing interests.

\section{References}

1. Siegel RL, Miller KD and Jemal A: Cancer statistics, 2020. CA Cancer J Clin 70: 7-30, 2020.

2. Rahib L, Smith BD, Aizenberg R, Rosenzweig AB, Fleshman JM and Matrisian LM: Projecting cancer incidence and deaths to 2030: The unexpected burden of thyroid, liver, and pancreas cancers in the United States. Cancer Res 74: 2913-2921, 2014.

3. Kamisawa T, Wood LD, Itoi T and Takaori K: Pancreatic cancer. Lancet 388: 73-85, 2016.

4. Zhang X, Shi S, Zhang B, Ni Q, Yu X and Xu J: Circulating biomarkers for early diagnosis of pancreatic cancer: Facts and hopes. Am J Cancer Res 8: 332-353, 2018.

5. Venkitaraman AR: Cancer susceptibility and the functions of BRCA1 and BRCA2. Cell 108: 171-182, 2002.

6. Huang L, Wu C, Yu D, Wang C, Che X, Miao X, Zhai K, Chang J, Jiang G, Yang X, et al: Identification of common variants in BRCA2 and MAP2K4 for susceptibility to sporadic pancreatic cancer. Carcinogenesis 34: 1001-1015, 2013.

7. Sikdar N, Saha G, Dutta A, Ghosh S, Shrikhande SV and Banerjee S: Genetic alterations of periampullary and pancreatic ductal adenocarcinoma: An overview. Curr Genomics 19: 444-463, 2018.

8. Zhang F, Ma J, Wu J, Ye L, Cai H, Xia B and Yu X: PALB2 links BRCA1 and BRCA2 in the DNA-damage response. Curr Biol 19: 524-529, 2009.

9. Reid S, Schindler D, Hanenberg H, Barker K, Hanks S, Kalb R, Neveling K, Kelly P, Seal S, Freund M, et al: Biallelic mutations in PALB2 cause Fanconi anemia subtype FA-N and predispose to childhood cancer. Nat Genet 39: 162-164, 2007.

10. Xia B, Dorsman JC, Ameziane N, de Vries Y, Rooimans MA, Sheng Q, Pals G, Errami A, Gluckman E, Llera J, et al: Fanconi anemia is associated with a defect in the BRCA2 partner PALB2. Nat Genet 39: 159-161, 2007.

11. Chen P, Liang J, Wang Z, Zhou X, Chen L, Li M, Xie D, Hu Z, Shen $\mathrm{H}$ and Wang H: Association of common PALB2 polymorphisms with breast cancer risk: A case-control study. Clin Cancer Res 14: 5931-5937, 2008.

12. Southey MC, Teo ZL and Winship I: PALB2 and breast cancer: Ready for clinical translation! Appl Clin Genet 6: 43-52, 2013.

13. Borecka M, Zemankova P, Vocka M, Soucek P, Soukupova J, Kleiblova P, Sevcik J, Kleibl Z and Janatova M: Mutation analysis of the PALB2 gene in unselected pancreatic cancer patients in the Czech Republic. Cancer Genet 209: 199-204, 2016.

14. Blanco A, de la Hoya M, Osorio A, Diez O, Miramar MD, Infante M, Martinez-Bouzas C, Torres A, Lasa A, Llort G, et al: Analysis of PALB2 gene in BRCA1/BRCA2 negative Spanish hereditary breast/ovarian cancer families with pancreatic cancer cases. PLoS One 8: e67538, 2013.

15. Harinck F, Kluijt I, van Mil SE, Waisfisz Q, van Os TA, Aalfs CM, Wagner A, Olderode-Berends M, Sijmons RH, Kuipers EJ, et al: Routine testing for PALB2 mutations in familial pancreatic cancer families and breast cancer families with pancreatic cancer is not indicated. Eur J Hum Genet 20: 577-579, 2012.

16. Hofstatter EW, Domchek SM, Miron A, Garber J, Wang M, Componeschi K, Boghossian L, Miron PL, Nathanson KL and Tung N: PALB2 mutations in familial breast and pancreatic cancer. Fam Cancer 10: 225-231, 2011.

17. Takeuchi S, Doi M, Ikari N, Yamamoto $M$ and Furukawa T: Mutations in BRCA1, BRCA2, and PALB2, and a panel of 50 cancer-associated genes in pancreatic ductal adenocarcinoma. Sci Rep 8: 8105, 2018.

18. Waddell N, Pajic M, Patch AM, Chang DK, Kassahn KS, Bailey P, Johns AL, Miller D, Nones K, Quek K, et al: Whole genomes redefine the mutational landscape of pancreatic cancer. Nature 518: 495-501, 2015.

19. Golan T, Kanji ZS, Epelbaum R, Devaud N, Dagan E, Holter S, Aderka D, Paluch-Shimon S, Kaufman B, Gershoni-Baruch R, et al: Overall survival and clinical characteristics of pancreatic cancer in BRCA mutation carriers. $\mathrm{Br}$ J Cancer 111: 1132-1138, 2014.

20. Wattenberg MM, Asch D, Yu S, O'Dwyer PJ, Domchek SM, Nathanson KL, Rosen MA, Beatty GL, Siegelman ES and Reiss KA: Platinum response characteristics of patients with pancreatic ductal adenocarcinoma and a germline BRCA1, BRCA2 or PALB2 mutation. Br J Cancer 122: 333-339, 2020.

21. Dell'Aquila E, Fulgenzi CAM, Minelli A, Citarella F, Stellato M, Pantano F, Russano M, Cursano MC, Napolitano A, Zeppola T, et al: Prognostic and predictive factors in pancreatic cancer. Oncotarget 11: 924-941, 2020 
22. Edge S, Byrd DR, Compton CC, Fritz AG, Greene FL and Trotti A AJCC Cancer Staging Manual. 7th edition. Springer-Verlag, New York, NY, 2009.

23. Livak KJ and Schmittgen TD: Analysis of relative gene expression data using real-time quantitative PCR and the 2(-Delta Delta C(T)) method. Methods 25: 402-408, 2001.

24. Siegel RL, Miller KD and Jemal A: Cancer statistics, 2019. CA Cancer J Clin 69: 7-34, 2019.

25. Ducy M, Sesma-Sanz L, Guitton-Sert L, Lashgari A, Gao Y, Brahiti N, Rodrigue A, Margaillan G, Caron MC, Côté J, et al: The tumor suppressor PALB2: Inside out. Trends Biochem Sci 44: 226-240, 2019.

26. Sy SM, Huen MS and Chen J: PALB2 is an integral component of the BRCA complex required for homologous recombination repair. Proc Natl Acad Sci USA 106: 7155-7160, 2009

27. Yang G, Mercado-Uribe I, Multani AS, Sen S, Shih Ie M, Wong KK, Gershenson DM and Liu J: RAS promotes tumorigenesis through genomic instability induced by imbalanced expression of Aurora-A and BRCA2 in midbody during cytokinesis. Int J Cancer 133: 275-285, 2013.

28. Egawa C, Miyoshi Y, Taguchi T, Tamaki Y and Noguchi S: High BRCA2 mRNA expression predicts poor prognosis in breast cancer patients. Int J Cancer 98: 879-882, 2002.

29. Li J, Li M, Chen P and Ba Q: High expression of PALB2 predicts poor prognosis in patients with advanced breast cancer. FEBS Open Bio 8: 56-63, 2017.

30. Poumpouridou N, Acha-Sagredo A, Goutas N, Vlachodimitropoulos D, Chatziioannidou I, Lianidou E, Liloglou $\mathrm{T}$ and Kroupis C: Development and validation of molecular methodologies to assess PALB2 expression in sporadic breast cancer. Clin Biochem 49: 253-259, 2016.

31. Bleuyard JY, Butler RM and Esashi F: Perturbation of PALB2 function by the T413S mutation found in small cell lung cancer. Wellcome Open Res 2: 110, 2017.

32. Nakagomi H, Sakamoto I, Hirotsu Y, Amemiya K, Mochiduki H and Omata M: Analysis of PALB2 mutations in 155 Japanese patients with breast and/or ovarian cancer. Int J Clin Oncol 21: 270-275, 2016.

33. Rahman N, Seal S, Thompson D, Kelly P, Renwick A, Elliott A, Reid S, Spanova K, Barfoot R, Chagtai T, et al: PALB2, which encodes a BRCA2-interacting protein, is a breast cancer susceptibility gene. Nat Genet 39: 165-167, 2007.
34. Casadei S, Norquist BM, Walsh T, Stray S, Mandell JB, Lee MK Stamatoyannopoulos JA and King MC: Contribution of inherited mutations in the BRCA2-interacting protein PALB2 to familial breast cancer. Cancer Res 71: 2222-2229, 2011.

35. Zhu Y, Zhai K, Ke J, Li J, Gong Y, Yang Y, Tian J, Zhang Y, Zou D, Peng X, et al: BRCA1 missense polymorphisms are associated with poor prognosis of pancreatic cancer patients in a Chinese population. Oncotarget 8: 36033-36039, 2017.

36. Jones S, Hruban RH, Kamiyama M, Borges M, Zhang X, Parsons DW, Lin JC, Palmisano E, Brune K, Jaffee EM, et al: Exomic sequencing identifies PALB2 as a pancreatic cancer susceptibility gene. Science 324: 217, 2009.

37. Slater EP, Langer P, Niemczyk E, Strauch K, Butler J, Habbe N, Neoptolemos JP, Greenhalf W and Bartsch DK: PALB2 mutations in European familial pancreatic cancer families. Clin Genet 78: 490-494, 2010.

38. Giovannetti E, van der Borden CL, Frampton AE, Ali A, Firuzi O and Peters GJ: Never let it go: Stopping key mechanisms underlying metastasis to fight pancreatic cancer. Semin Cancer Biol 44: 43-59, 2017.

39. Garces-Descovich A, Beker K, Jaramillo-Cardoso A, James Moser A and Mortele KJ: Applicability of current NCCN Guidelines for pancreatic adenocarcinoma resectability: Analysis and pitfalls. Abdom Radiol (NY) 43: 314-322, 2018.

40. Lefebvre C, Bachelot T, Filleron T, Pedrero M, Campone M, Soria JC, Massard C, Lévy C, Arnedos M, Lacroix-Triki M, et al: Mutational profile of metastatic breast cancers: A retrospective analysis. PLoS Med 13: e1002201, 2016.

41. Pritchard CC, Mateo J, Walsh MF, De Sarkar N, Abida W, Beltran H, Garofalo A, Gulati R, Carreira S, Eeles R, et al: Inherited DNA-Repair gene mutations in men with metastatic prostate cancer. New Engl J Med 375: 443-453, 2016.

c) (i) $\Theta$ This work is licensed under a Creative Commons c) Attribution-NonCommercial-NoDerivatives 4.0 International (CC BY-NC-ND 4.0) License. 